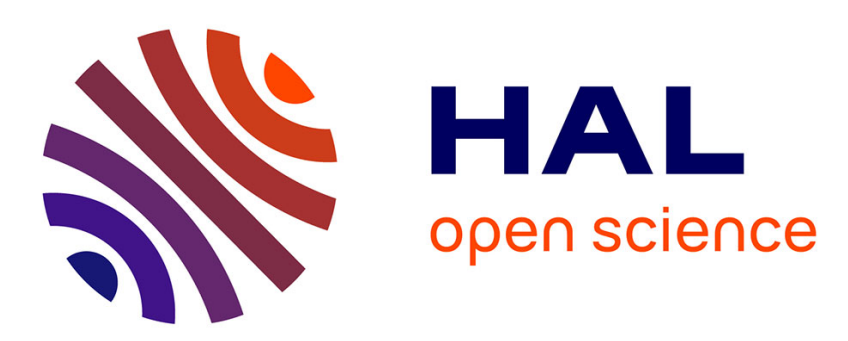

\title{
Enhanced electrical conductivity and photoconductive properties of Sn-doped Sb2Se3 crystals
}

S. Chen, X. Qiao, Z. Zheng, M. Cathelinaud, Hongli Ma, X. Fan, Xianghua Zhang

\section{> To cite this version:}

S. Chen, X. Qiao, Z. Zheng, M. Cathelinaud, Hongli Ma, et al.. Enhanced electrical conductivity and photoconductive properties of Sn-doped Sb2Se3 crystals. Journal of Materials Chemistry C, 2018, 6 (24), pp.6465-6470. 10.1039/c8tc01683f . hal-01835068

HAL Id: hal-01835068

https://hal-univ-rennes1.archives-ouvertes.fr/hal-01835068

Submitted on 3 Sep 2018

HAL is a multi-disciplinary open access archive for the deposit and dissemination of scientific research documents, whether they are published or not. The documents may come from teaching and research institutions in France or abroad, or from public or private research centers.
L'archive ouverte pluridisciplinaire HAL, est destinée au dépôt et à la diffusion de documents scientifiques de niveau recherche, publiés ou non, émanant des établissements d'enseignement et de recherche français ou étrangers, des laboratoires publics ou privés. 


\title{
Enhanced electrical conductivity and photoconductive properties of $\mathrm{Sn}$-doped $\mathrm{Sb}_{2} \mathrm{Se}_{3}$ crystals $\uparrow$
}

\author{
Shuo Chen, ab Xvsheng Qiao, a Zhuanghao Zheng, ${ }^{\mathrm{b}}$ Michel Cathelinaud, ${ }^{\mathrm{b}}$ \\ Hongli Ma, ${ }^{b}$ Xianping Fan ${ }^{* a}$ and Xianghua Zhang ${ }^{* b}$
}

$\mathrm{Sb}_{2} \mathrm{Se}_{3}$ is a highly interesting semiconductor with high absorption coefficient in the visible range and is composed of non-toxic and earth-abundant elements. To overcome the challenge of intrinsic low electrical conductivity of $\mathrm{Sb}_{2} \mathrm{Se}_{3}$ crystals, tin-doped $\left(\mathrm{Sn}_{x} \mathrm{Sb}_{1-x}\right)_{2} \mathrm{Se}_{3}$ semiconductors $(x=0.00,0.03,0.05$, 0.07 and 0.10 ) have been synthesized by a conventional melt-quenching method in a vacuum sealed silica tube. With increasing $\mathrm{Sn}$ doping concentration, the $\left(\mathrm{Sn}_{x} \mathrm{Sb}_{1-x}\right)_{2} \mathrm{Se}_{3}$ crystals exhibited a great improvement in electrical conductivity by several orders of magnitude thanks to the great increase of carrier concentration reaching almost $2 \times 10^{16} \mathrm{~cm}^{-3}$. Compared to undoped $\mathrm{Sb}_{2} \mathrm{Se}_{3}$, the dark current density of a representative $\left(\mathrm{Sn}_{0.10} \mathrm{Sb}_{0.90}\right)_{2} \mathrm{Se}_{3}$ increased by approximately 10 times and the photocurrent density with essentially visible illumination increased by approximately 14 times. In addition, the doped sample showed a faster, reversible and stable photoresponse. These excellent performances combined with a simple and easily scalable synthesis method pave the way for using this semiconductor for highly efficient photoelectric devices.

\section{Introduction}

Antimony selenide $\left(\mathrm{Sb}_{2} \mathrm{Se}_{3}\right)$ is an important V-VI binary semiconductor with excellent photovoltaic, photoconducting, photocatalytic and thermoelectric properties. ${ }^{1-6}$ It has a high absorption coefficient higher than $10^{5} \mathrm{~cm}^{-1}$ in the visible region and an optimum bandgap width of around $1.1 \mathrm{eV}$ for single junction solar cells. In addition, it is stable and nontoxic and composed of earth abundant elements. These properties

40 make it a promising candidate for various important applications in diverse areas such as solar energy conversion, photodetector technology, thermoelectric power generation, and opto-electronics in the near-infrared region. ${ }^{5,7-11}$

Despite the important research efforts, the progress in application development is still limited. One major issue with $\mathrm{Sb}_{2} \mathrm{Se}_{3}$ is its intrinsic low electrical conductivity $\left(10^{-6} \Omega^{-1} \mathrm{~m}^{-1}\right.$, in the bulk state), which results in low thermoelectric power factor, low spectral response and low external quantum efficiency. ${ }^{12,13}$ A few studies to improve the electrical

\footnotetext{
${ }^{a}$ State Key Laboratory of Silicon Materials, School of Materials Science and Engineering, Zhejiang University, Hangzhou, 310027, P. R. China. E-mail:fanxp@zju.edu.cn

${ }^{b}$ Univ Rennes, CNRS, ISCR UMR6226, 35000 Rennes, France. E-mail: xzhang@univ-rennes1.fr

$55 \dagger$ Electronic supplementary information (ESI) available. See DOI: 10.1039/ c8tc01683f
}

conductivity of $\mathrm{Sb}_{2} \mathrm{Se}_{3}$ have been reported. For example, Choi et al. developed $\mathrm{Sb}_{2} \mathrm{Se}_{3}$ nanowires decorated with $\mathrm{Ag}_{2} \mathrm{Se}$ nanoparticles, which increased the electrical conductivity of the $\mathrm{Sb}_{2} \mathrm{Se}_{3}$ nanowires. ${ }^{12}$ Zhang et al. explained an interpenetrating iodine-doped- $\mathrm{Sb}_{2} \mathrm{Se}_{3} / \mathrm{Cu}_{2} \mathrm{GeSe}_{3}$ heterojunction network fabricated by controlling the crystallization of chalcogenide glass which can improve the photoelectric performance. ${ }^{14}$ Our previous work including the synthesis of hybrid nanorods with the $\mathrm{Sb}_{2} \mathrm{Se}_{3} / \mathrm{AgSbSe}{ }_{2}$ heterojunction structure also shows a higher electrical conductivity and photodetector performance. ${ }^{10}$ Despite these endeavors, the synthesis of $\mathrm{Sb}_{2} \mathrm{Se}_{3}$-based crystals with controllable electrical conductivity has been rarely reported and still needs further exploration.

Doping is an efficient way to tune the electrical and photoelectrical performances of semiconductors. It has been widely utilized in the semiconductor industry. ${ }^{15-17}$ For example, S-doped $\mathrm{In}_{2} \mathrm{Se}_{3}$ nanowires show a much higher conductivity and spectral responsivity to visible light than undoped $\mathrm{In}_{2} \mathrm{Se}_{3}$ nanowires. ${ }^{17}$ A small amount of Sn leads to a significant increase in the electrical conductivity and photoconductivity of the amorphous $\mathrm{As}_{2} \mathrm{Se}_{3}$ films. ${ }^{18}$ Herein, we demonstrate a conventional melt-quenching method to controllably synthesize high-quality and variably doped $\left(\mathrm{Sn}_{x} \mathrm{Sb}_{1-x}\right)_{2} \mathrm{Se}_{3}$ semiconducting crystals with the Sn ratio $x$ ranging from 0.00 to 0.10 . By incorporating additional $\mathrm{Sn}$ atoms into the binary $\mathrm{Sb}_{2} \mathrm{Se}_{3}$, a significant improvement in the electrical conductivity and 
1 photoconductivity is observed. Moreover, the as-synthesized bulk samples with easy and relatively large-scale synthesis features show very interesting properties for fabricating $\mathrm{Sb}_{2} \mathrm{Se}_{3}$-based photoelectric devices.

\section{Experimental}

\section{Synthesis of $\left(\mathrm{Sn}_{x} \mathrm{Sb}_{1-x}\right)_{2} \mathrm{Se}_{3}$ crystals}

A conventional melt-quenching method using high purity tin (99.99\%), antimony (99.999\%) and selenium (99.999\%) as raw materials was used to synthesize $\left(\mathrm{Sn}_{x} \mathrm{Sb}_{1-x}\right)_{2} \mathrm{Se}_{3}$ crystals. A schematic illustration of the preparation process is shown in Fig. S1 (ESI $\dagger$ ). At first, a mixture of $12 \mathrm{~g}$ of raw materials according to the chemical composition $\left(\mathrm{Sn}_{x} \mathrm{Sb}_{1-x}\right)_{2} \mathrm{Se}_{3}$ (where $x=0.00,0.03,0.05,0.07$ and 0.10 , respectively) was loaded into a fully cleaned silica tube of $1 \mathrm{~cm}$ internal diameter. A vacuum trap that was placed in liquid nitrogen was used to condense any volatile products and also to improve the vacuum. The silica tube was sealed under a typical vacuum of around $10^{-5}$ mbar and then was placed in a rocking furnace. The mixture was heated to $700{ }^{\circ} \mathrm{C}$ at a rate of $1.5{ }^{\circ} \mathrm{C} \mathrm{min}{ }^{-1}$ and held for about 8 hours to ensure complete melting and homogenization. Then it was cooled down to $650{ }^{\circ} \mathrm{C}$ during 2 hours and subsequent quenching in water at room temperature. Finally, in order to reduce the stress generated from the thermal shock in the quenching process, the as-synthesized crystals were annealed at $350{ }^{\circ} \mathrm{C}$ for 3 hours.

\section{Characterization}

X-ray diffraction (XRD) analysis was carried out using a PANalytical's X-ray diffractometer with a step width of $0.02^{\circ}$ and a counting duration of $0.13 \mathrm{~s}$ per step. Scanning electron microscopy (SEM) images and the corresponding Energy Dispersive 5 Spectrometer (EDS) elemental mappings were obtained using a JEOL JSM-7100F thermal field emission electron microscope equipped with an EDAX instrument. TEM, HRTEM and SAED images were obtained using a JEOL JEM-2100 field emission transmission electron microscope. The bulk sample was milled

40 and then dissolved in chloroform before being placed on the surface of a copper grid for further TEM measurements. The quality and composition of the crystals were also analyzed by X-ray photoelectron spectroscopy (XPS, ESCALAB). Before electrical conductivity and photoconductivity measurements, the discs with a thickness of about $2 \mathrm{~mm}$ also with surface polishing. Electrical resistivity and bulk carrier density were measured using a van der Pauw Ecopia HMS-3000 Hall Measurement System (Ecopia Corporation, Anyang, South by the conventional four-probe method in the temperature range of 300-400 K. To characterize the photoelectric performance, the photo-electro-chemical (PEC) measurement has been carried out using the classical 3-electrode method, where $\mathrm{Ag} / \mathrm{AgCl}$ in saturated $\mathrm{KCl}$ was used as the reference electrode, Pt-wire as the counter electrode and our sample as the working electrode. Additionally, to prepare the working electrode, the as-synthesized bulk $\left(\mathrm{Sn}_{x} \mathrm{Sb}_{1-x}\right)_{2} \mathrm{Se}_{3}$ crystals were sliced into small discs with a diameter of $10 \mathrm{~mm}$ and a thickness of about $2 \mathrm{~mm}$, and then the surface was finely polished and cleaned. After this, silver paint was homogeneously applied on one face of the disc-like sample, acting as the back contact, which was connected by a copper wire and then covered with a layer of epoxy resin to be isolated from the electrolyte during the PEC test. Then these electrodes were immersed into the electrolyte (0.5 mol L $\mathrm{L}^{-1} \mathrm{LiClO}_{4}$ solution), and a white light tungsten halogen lamp with controllable light intensities was used as the light source. A mechanical chopper was used to obtain chopped light and an Autolab Metrohm potentiostat was used to record the current-voltage characteristics.

\section{Results and discussion}

Fig. 1a shows the XRD patterns of the as-synthesized $\left(\mathrm{Sn}_{x} \mathrm{Sb}_{1-x}\right)_{2} \mathrm{Se}_{3}$ polycrystalline semiconductors $(x=0.00,0.03$, $0.05,0.07$ and 0.10 ) acquired on sliced and polished disc-like samples, which all exhibit prominent peaks in agreement with the JCPDS standard card (15-0861) of the orthorhombic phase of $\mathrm{Sb}_{2} \mathrm{Se}_{3}$ without a second phase, indicating the absence of any detectable impurity. The absence of multiple sets of peaks also indicates that the $\left(\mathrm{Sn}_{x} \mathrm{Sb}_{1-x}\right)_{2} \mathrm{Se}_{3}$ crystals do not contain a second crystalline phase after Sn doping. Then the XRD patterns of the corresponding powdered samples obtained by grinding the bulk crystals into fine powders can be indexed to the same crystalline phase, as shown in Fig. 1b, suggesting that the crystallization process takes place uniformly inside the bulk sample and is not restricted to the surface. The relative peak intensity is different between the powder and the bulk because the preferential orientation in the bulk is destroyed during the grinding of the powders. It is noted that further
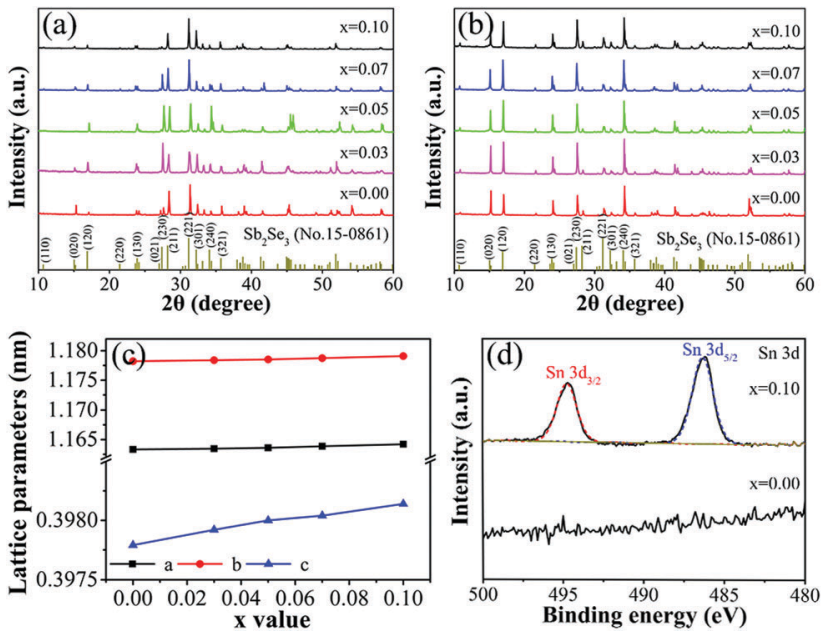

Fig. 1 (a) XRD patterns of the bulk $\left(\mathrm{Sn}_{x} \mathrm{Sb}_{1-x}\right)_{2} \mathrm{Se}_{3}$ crystals $(x=0.00,0.03$ $0.05,0.07$ and 0.10$)$. (b) XRD patterns of the powdered $\left(\mathrm{Sn}_{x} \mathrm{Sb}_{1-x}\right)_{2} \mathrm{Se}_{3}$ samples. (c) Lattice constants $a, b$ and $c$ derived from powder XRD peaks, plotted as a function of $S n$ doping concentration ( $x$ value). (d) XPS spectrum of $\mathrm{Sn}-3 \mathrm{~d}$ for the $\left(\mathrm{Sn}_{x} \mathrm{Sb}_{1-x}\right)_{2} \mathrm{Se}_{3}$ crystals $(x=0.00$ and 0.10 ).
45 
1 increase of Sn doping concentration will lead to additional diffraction peaks corresponding to orthorhombic $\mathrm{Sn}_{2} \mathrm{Sb}_{4} \mathrm{Se}_{8}$ (JCPDS Card No. 36-1206), indicating the existence of the second phase (Fig. S2, ESI $\dagger$ ). The lattice parameters $a, b$ and $c$ were determined by refining the diffraction patterns through a Rietveld method implemented in the FULLPROF program, ${ }^{19}$ which show a slight increase upon increasing the Sn doping concentration (Fig. 1c). This can be attributed to the small difference in the atomic radius between $\mathrm{Sb}(0.160 \mathrm{~nm})$ and $\mathrm{Sn}$ $(0.158 \mathrm{~nm})$. And this slight difference satisfies the criterion for substitution solubility proposed by Hume-Rothery who pointed out that an extensive solid solubility of one metal in another only occurs if the diameter of the atoms differs by less than $15 \%{ }^{20}$ Furthermore, the calculated unit cell volume of the

$15\left(\mathrm{Sn}_{x} \mathrm{Sb}_{1-x}\right)_{2} \mathrm{Se}_{3}$ crystals gradually increases from $545.23 \AA^{3}$ $(x=0.00)$ to $546.53 \AA^{3}(x=0.10)$, and this increase is known to occur due to doping. ${ }^{21}$ Fig. $1 \mathrm{~d}$ shows the XPS spectrum of Sn$3 \mathrm{~d}$, compared to the pure $\mathrm{Sb}_{2} \mathrm{Se}_{3}$. Two obvious peaks located at 494.7 and $486.2 \mathrm{eV}$ from $\left(\mathrm{Sn}_{0.10} \mathrm{Sb}_{0.90}\right)_{2} \mathrm{Se}_{3}$ crystals indicate the existence of $\mathrm{Sn}$, further confirming the successful doping.

The $\left(\mathrm{Sn}_{x} \mathrm{Sb}_{1-x}\right)_{2} \mathrm{Se}_{3}$ crystals' $(x=0.00,0.03,0.05,0.07$ and 0.10$)$ morphology of the freshly fractured surface observed by SEM is shown in Fig. S3 (ESI $\dagger$ ). All the samples consisted of large crystals with a layered-like structure and clear strips, indicating a preferential orientation. According to the theoretical and experimental studies, bulk $\mathrm{Sb}_{2} \mathrm{Se}_{3}$ has an orthorhombic crystal structure and is composed of $1 \mathrm{D}$ ribbon-like $\left(\mathrm{Sb}_{4} \mathrm{Se}_{6}\right)_{n}$ units stacking together via van der Waals interactions. ${ }^{22}$ Its preferential growth is indeed determined intrinsically by the anisotropic $\mathrm{Sb}-\mathrm{Se}$ atom chain or the layer structure. ${ }^{7,10}$ To further investigate the doping nature and also the grain size of the $\left(\mathrm{Sn}_{x} \mathrm{Sb}_{1-x}\right)_{2} \mathrm{Se}_{3}$ crystals, the finely polished disk-like samples were etched chemically by $1 \mathrm{~mol} \mathrm{~L}^{-1}$ $\mathrm{NaOH}$ solution for $20 \mathrm{~min}$ at room temperature. As shown in Fig. 2a, the $\mathrm{Sb}_{2} \mathrm{Se}_{3}$ crystal consists of some big micron-sized grains. Fig. $2 \mathrm{~b}$ shows a representative etched microstructure of the $\left(\mathrm{Sn}_{0.05} \mathrm{Sb}_{0.95}\right)_{2} \mathrm{Se}_{3}$ crystal; the grains are clearly shown with an average size of approximately $15 \mu \mathrm{m}$. In addition, compositional spatial distribution was determined by EDS elemental mappings marked with $\mathrm{Sn}, \mathrm{Sb}$ and $\mathrm{Se}$, which reveals a uniform distribution between the grain boundaries can also be observed after chemical etching. Then for the $\left(\mathrm{Sn}_{0.10} \mathrm{Sb}_{0.90}\right)_{2} \mathrm{Se}_{3}$ crystal with a higher $\mathrm{Sn}$ doping concentration, more obvious Sn-rich regions can be observed, as shown in Fig. 2c. This could be explained by phase separation, is reasonable to occur, the minor Sn-rich phase solidifies first, and then is pushed to the grain boundaries upon freezing of the major phase. To further reveal the elemental composition of these Sn-rich regions, EDS analysis has been performed. Fig. 2d shows the EDS spectra recorded from two different positions (Point 1 in Fig. 2b and Point 2 in Fig. 2c); the atomic ratio of $\mathrm{Sn}: \mathrm{Sb}:$ Se is $14.54: 23.74: 61.72$ and $15.39: 22.22: 62.39$ for Point 1 and Point 2, respectively. It is noted that the composition analysis of these Sn-rich regions performed on different $\left(\mathrm{Sn}_{x} \mathrm{Sb}_{1-x}\right)_{2} \mathrm{Se}_{3}$ crystals show quite similar results, close to the ratio of $2: 4: 8$ for $\mathrm{Sn}_{2} \mathrm{Sb}_{4} \mathrm{Se}_{8}$, which is also consistent with the XRD results.
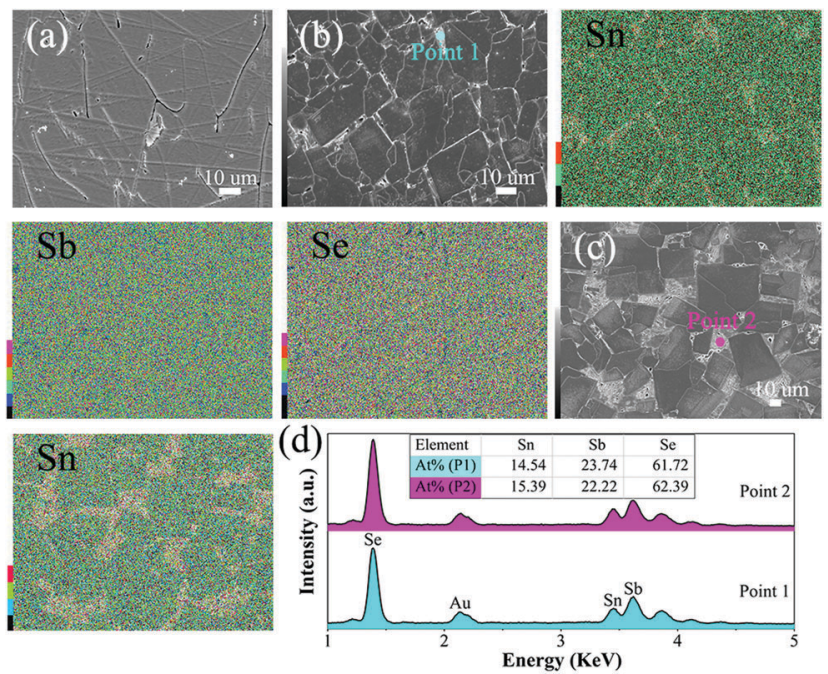

10

Fig. 2 SEM images of (a) the $\mathrm{Sb}_{2} \mathrm{Se}_{3}$ crystal, (b) the $\left(\mathrm{Sn}_{0.05} \mathrm{Sb}_{0.95}\right)_{2} \mathrm{Se}_{3}$ crystal and (c) the $\left(\mathrm{Sn}_{0.10} \mathrm{Sb}_{0.90}\right)_{2} \mathrm{Se}_{3}$ crystal surface with chemical etching by $\mathrm{NaOH}$ solution and following the corresponding EDS elemental mappings of Sn, Sb and Se. (d) EDS spectra recorded from two different positions (Point 1 in (b) and Point 2 in (c)).
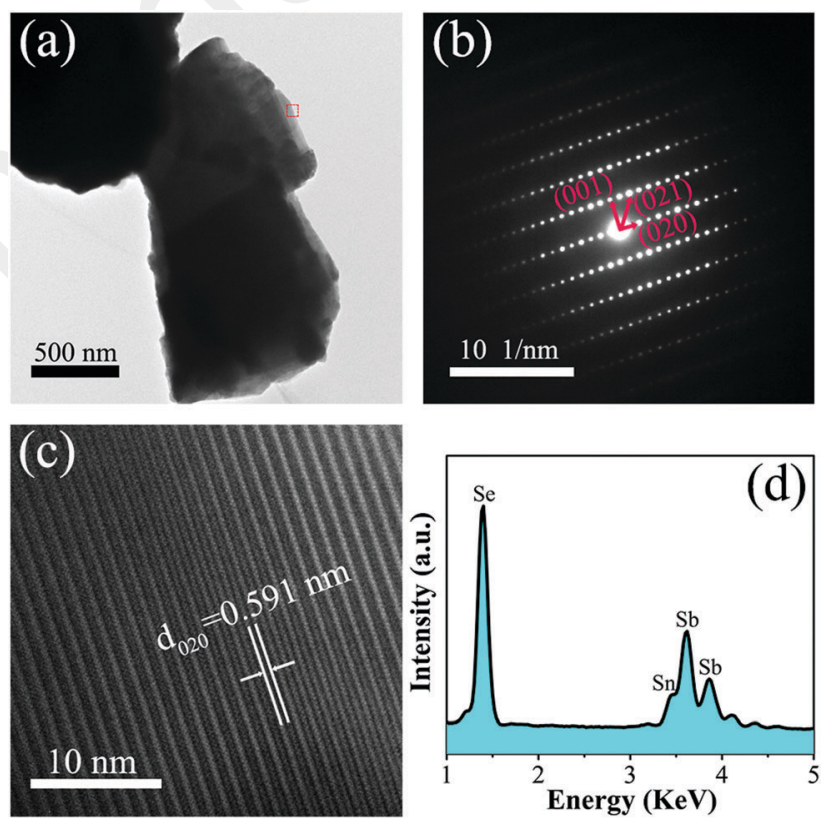

Fig. 3 (a) TEM image of the representative $\left(\mathrm{Sn}_{0.10} \mathrm{Sb}_{0.90}\right)_{2} \mathrm{Se}_{3}$ crystal. SAED pattern (b) and the corresponding HRTEM image (c) from the rectangle marked area in (a). (d) EDS spectrum.

Fig. 3a shows the TEM image of an isolated particle milled from the $\left(\mathrm{Sn}_{0.10} \mathrm{Sb}_{0.90}\right)_{2} \mathrm{Se}_{3}$ crystal. The selected area electron diffraction (SAED) pattern (Fig. 3b) from the red marked area (in Fig. 3a) is a periodic spot pattern, suggesting its highly crystalline nature. Fig. 3c shows the corresponding HRTEM image with obvious lattice fringes associated with the (020) planes ( $d$-spacing of $0.591 \mathrm{~nm}$ ) of the orthorhombic phase $\mathrm{Sb}_{2} \mathrm{Se}_{3}$. Then the EDS spectrum (Fig. 3d) exhibits strong $\mathrm{Sb}$

1

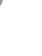


1 and Se peaks, and also an observable Sn peak located at $3.44 \mathrm{eV}$, indicating the successful Sn doping in this crystal.

Fig. 4a shows the electrical resistivity of the as-synthesized $\left(\mathrm{Sn}_{x} \mathrm{Sb}_{1-x}\right)_{2} \mathrm{Se}_{3}$ crystals with different $x$ values. It can be seen that the pure $\mathrm{Sb}_{2} \mathrm{Se}_{3}$ exhibits extremely high resistivity. With $\mathrm{Sn}$ doping, a significant decrease of resistivity is observed, and the decrease is almost exponential with the $x$ value. The temperature dependent electrical conductivity $(\sigma)$ in the temperature $(T)$ range of 300-400 $\mathrm{K}$ has also been studied. As shown in Fig. 4b, plots of $\ln \sigma$ versus $1 / T$ indicate that the electrical conductivity increases with increasing temperature for all samples, confirming semiconductor characteristics of these samples. Moreover, the conductivity exhibits single activation energy in the temperature range of measurement, according to the Arrhenius equation:

$$
\sigma=\sigma_{0} \exp (-\Delta E / k T)
$$

where $\sigma_{0}$ is the pre-exponential factor, $\Delta E$ is the activation energy and $k$ is the Boltzmann constant. ${ }^{23}$ Thus the thermal activation energy $\Delta E$ can be calculated from the slope, while the intercept on the $y$-axis gives the $\sigma_{0}$ value. Then the bulk carrier density and the doping type were determined by Hall measurements. All the electrical parameters of $\left(\mathrm{Sn}_{x} \mathrm{Sb}_{1-x}\right)_{2} \mathrm{Se}_{3}$ crystals are summarized in Table 1. It is obvious that the room temperature electrical conductivity shows an increase with several orders of magnitude after Sn doping, accompanied by a synchronous increase of bulk carrier density, which change from $1.44 \times 10^{11} \mathrm{~cm}^{-3}(x=0.00)$ to $1.94 \times 10^{16} \mathrm{~cm}^{-3}(x=0.10)$. Importantly, such a great improvement will provide a bright

future for widening applications of $\mathrm{Sb}_{2} \mathrm{Se}_{3}$. Since the activation energy varies inversely with the charge carrier density, Sn incorporation in this system should theoretically lead to a decrease of activation energy, which is consistent with the calculated $\Delta E$ values. The pre-exponential factor has a close relationship with the defect or impurities in the crystals, Sn doping into $\mathrm{Sb}_{2} \mathrm{Se}_{3}$ also creates a compositional and configuration disorder, leading to an increase in the localized state density, which is an important factor for the change of $\sigma_{0}$ values. Table 1 also shows the conduction type of $\left(\mathrm{Sn}_{x} \mathrm{Sb}_{1-x}\right)_{2} \mathrm{Se}_{3}$ crystals; $\mathrm{Sn}$ incorporation has been found to result in P-type behavior, suggesting Sn substitution at the Sb site. Sn possesses one fewer valence electron than $\mathrm{Sb}$, thus replacing $\mathrm{Sb}$ with $\mathrm{Sn}$ constitutes hole doping. ${ }^{24}$
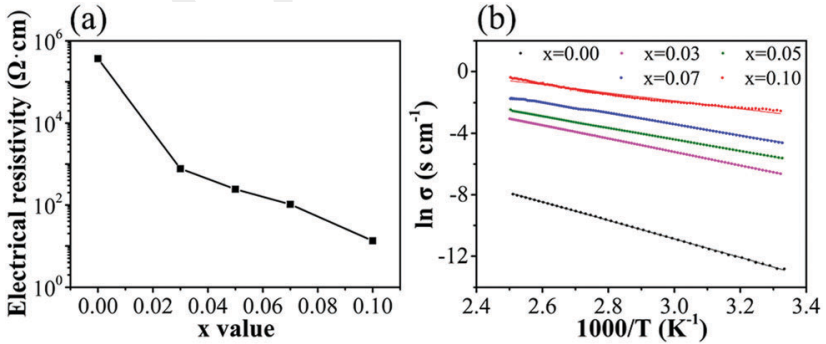

Fig. 4 (a) Electrical resistivity of $\left(\mathrm{Sn}_{x} \mathrm{Sb}_{1-x}\right)_{2} \mathrm{Se}_{3}$ crystals with different $x$ values. (b) Temperature dependent electrical conductivity $(\sigma)$ of $\left(\mathrm{Sn}_{x} \mathrm{Sb}_{1-x}\right)_{2} \mathrm{Se}_{3}$ crystals in the temperature $(T)$ range $300-400 \mathrm{~K}$.
Table 1 A summary of electrical conductivity $(\sigma)$, bulk carrier density $(n)$, pre-exponential factor $\left(\sigma_{0}\right)$, activation energy $(\Delta E)$ and conduction type of $\left(\mathrm{Sn}_{x} \mathrm{Sb}_{1-x}\right)_{2} \mathrm{Se}_{3}$ crystals with varied $x$

\begin{tabular}{llllll}
$x$ value & $\sigma\left(\mathrm{S} \mathrm{cm}^{-1}\right)$ & $n\left(\mathrm{~cm}^{-3}\right)$ & $\sigma_{0}\left(\mathrm{~S} \mathrm{~cm}^{-1}\right) \Delta E(\mathrm{eV})$ & Conduction type \\
\hline 0.00 & $2.71 \times 10^{-6} 1.44 \times 10^{11}$ & $1.31 \times 10^{3}$ & 0.52 & $\mathrm{~N}$ \\
0.03 & $1.30 \times 10^{-3}$ & $1.52 \times 10^{15}$ & $2.65 \times 10^{3}$ & 0.38 & $\mathrm{P}$ \\
0.05 & $4.12 \times 10^{-3}$ & $3.59 \times 10^{15}$ & $9.96 \times 10^{2}$ & 0.32 & $\mathrm{P}$ \\
0.07 & $9.65 \times 10^{-3}$ & $6.90 \times 10^{15}$ & $1.49 \times 10^{3}$ & 0.30 & $\mathrm{P}$ \\
0.10 & $7.50 \times 10^{-2}$ & $1.94 \times 10^{16}$ & $3.48 \times 10^{2}$ & 0.22 & $\mathrm{P}$
\end{tabular}

Photoconductivity is a well-known property of semiconductors which describes the electrical conductivity changes with the incident radiation. The photoconductive characteristics of the semiconducting $\left(\mathrm{Sn}_{x} \mathrm{Sb}_{1-x}\right)_{2} \mathrm{Se}_{3}$ crystals are investigated here by using the classical 3-electrode photo-electro-chemical (PEC) measurements. A schematic illustration of this PEC measurement is shown in Fig. 5a. It is noted that with this 3electrode technique which measures the generated and separated electron-hole pairs on the surface, it can eliminate the influence of the bulk resistance of the sample, facilitating therefore the comparison between different samples. Fig. 5b shows the current density versus voltage under chopped light using $\left(\mathrm{Sn}_{x} \mathrm{Sb}_{1-x}\right)_{2} \mathrm{Se}_{3}(x=0.00,0.03,0.05,0.07$ and 0.10$)$ as the working electrode, respectively. It is obvious that both dark current and photocurrent density of pure $\mathrm{Sb}_{2} \mathrm{Se}_{3}$ are very weak, which can be explained by its intrinsic low conductivity and low carrier concentration. For Sn-doped crystals, a significant increase of current density is observed, and this increase is proportional to the Sn doping concentration. Up to $x=0.10$, the dark current density increased approximately 10 times (from $-16 \mu \mathrm{A} \mathrm{cm}^{-2}$ to $-160 \mu \mathrm{A} \mathrm{cm}^{-2}$ at a bias voltage of $-0.6 \mathrm{~V}$ ) and the photocurrent density increased approximately 14 times

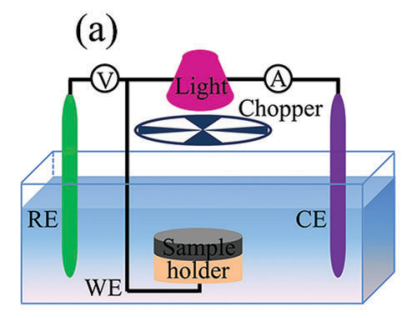

(c)

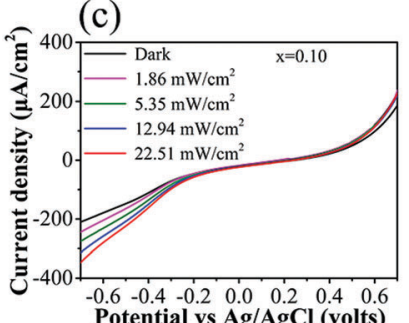

Potential vs Ag/AgCl (volts)
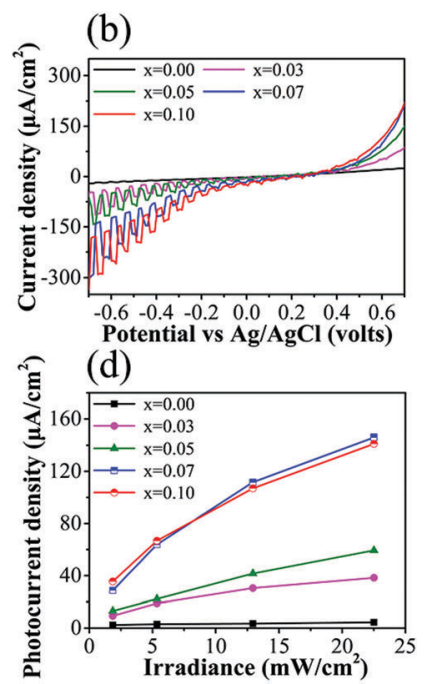

Fig. 5 (a) Schematic diagram of the 3-electrode photo-electro-chemical (PEC) measurement. (b) Current-voltage characteristics using $\left(\mathrm{Sn}_{x} \mathrm{Sb}_{1-x}\right)_{2} \mathrm{Se}_{3}$ crystals as working electrodes. (c) Dark current density and photocurrent densities at different power densities of the representative $\left(\mathrm{Sn}_{0.10} \mathrm{Sb}_{0.90}\right)_{2} \mathrm{Se}_{3}$ crystal. (d) Photocurrent densities measured as a function of incident light density at a bias voltage of $-0.7 \mathrm{~V}$. 
1 (from $-18 \mu \mathrm{A} \mathrm{cm}^{-2}$ to $-250 \mu \mathrm{A} \mathrm{cm}^{-2}$ at $-0.6 \mathrm{~V}$ ). This improvement can be considered as an important step to promote $\mathrm{Sb}_{2} \mathrm{Se}_{3}$-based semiconductors for application in high performance photoelectric devices. Fig. 5b also shows that the Sn-doped $\mathrm{Sb}_{2} \mathrm{Se}_{3}$ crystals are identified as p-type semiconductors, due to their photocurrent density increase with a negative bias, which is confirmed by the Hall measurement results. Then the light intensity dependence of current density is also investigated. As shown in Fig. 5c, using $\left(\mathrm{Sn}_{0.10} \mathrm{Sb}_{0.90}\right)_{2} \mathrm{Se}_{3}$ as an example, upon illumination, the current density is increased, and then it increases gradually with increasing light intensity. Additionally, current-voltage curves of $\left(\mathrm{Sn}_{x} \mathrm{Sb}_{1-x}\right)_{2} \mathrm{Se}_{3}$ at other $x$ values $(0.00,0.03,0.05$ and 0.07$)$ are shown in Fig. S4 (ESI $\dagger$ ), where similar phenomena were observed. All the samples show that the photocurrent strongly depends on the light intensity, which can be attributed to the change in photo-generated carriers. The corresponding photocurrent density as a function of light intensity is shown in Fig. $5 d$, and dependence can be described by the power law:

$$
J_{\mathrm{ph}}=A P^{\theta}
$$

where $J_{\mathrm{ph}}$ represents the photocurrent density; $P$ is the power density of the incident light; $A$ is a constant at a specific wavelength; and $\theta$ is an exponent. ${ }^{25,26}$ The fitting curves showed that $\theta=0.27,0.52,0.65,0.60$ and 0.54 for $x$ values of $0.00,0.03,0.05,0.07$ and 0.10 , respectively. All these $\theta$ values are non-unity, indicating that some surface defects and carrier trapping at the localized states could result in a complex electron-hole non-radiative recombination process. ${ }^{4}$

To evaluate the stability and the response speed of the photocurrent, chronoamperometry measurements have been performed. Fig. $6 \mathrm{a}$ and $\mathrm{b}$ show two plots of the time-resolved PEC response using $\mathrm{Sb}_{2} \mathrm{Se}_{3}$ and $\left(\mathrm{Sn}_{0.10} \mathrm{Sb}_{0.90}\right)_{2} \mathrm{Se}_{3}$ as the working electrode, respectively. As shown in Fig. 6a, when the light is (a)

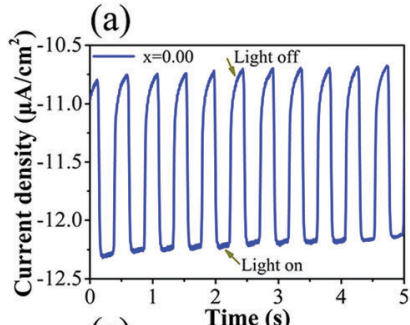

(c)

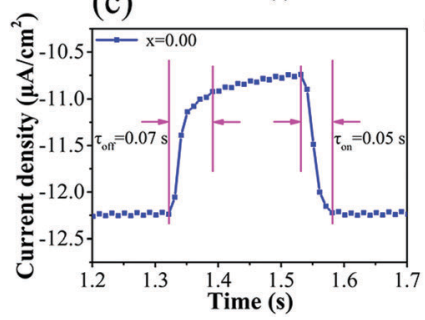

(b)

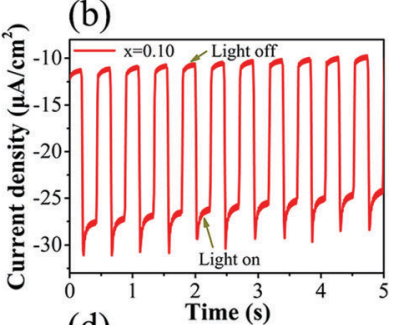

(d)

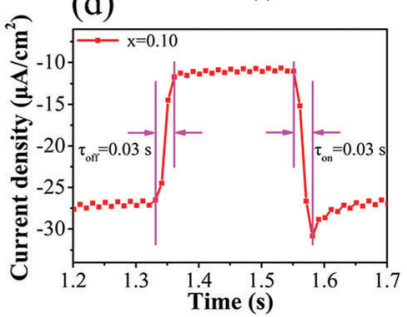

Fig. 6 Time-resolved PEC photoresponse by using $\mathrm{Sb}_{2} \mathrm{Se}_{3}$ (a) and $\left(\mathrm{Sn}_{0.10} \mathrm{Sb}_{0.90}\right)_{2} \mathrm{Se}_{3}$ (b) as the working electrode, respectively. (c) Magnified plot of one response cycle in (a), and (d) one response cycle in (b), used to obtain the response time and the recovery time. on, the current density rapidly increases from $-10.75 \mu \mathrm{A} \mathrm{cm}^{-2}$ (dark condition) to $-12.25 \mu \mathrm{A} \mathrm{cm}^{-2}$ (with $22.51 \mathrm{~mW} \mathrm{~cm}^{-2}$ light illumination), and then sharply returns to its initial value as the light is turned off. This light response remains the same after multiple cycles, indicating the excellent reversibility and stability of this $\mathrm{Sb}_{2} \mathrm{Se}_{3}$ PEC electrode. Then a similar photoresponse can be seen for $\left(\mathrm{Sn}_{0.10} \mathrm{Sb}_{0.90}\right)_{2} \mathrm{Se}_{3}$, except for a larger photocurrent density in the latter case (Fig. 6b). When using the $\left(\mathrm{Sn}_{0.10} \mathrm{Sb}_{0.90}\right)_{2} \mathrm{Se}_{3}$ crystal as the working electrode, a huge photocurrent density spike before arriving at a steady value can also be observed, which was caused by recombination in the presence of surface states. ${ }^{27}$ To extract the exact response time, one of the response periods is magnified, as shown in Fig. $6 \mathrm{c}$ and $\mathrm{d}$, respectively. Under closer examination, the response time $\left(\tau_{\text {on }}\right)$ and the recovery time $\left(\tau_{\text {off }}\right)$ of the $\mathrm{Sb}_{2} \mathrm{Se}_{3}$ working electrode are $0.05 \mathrm{~s}$ and $0.07 \mathrm{~s}$, respectively. For $\left(\mathrm{Sn}_{0.10} \mathrm{Sb}_{0.90}\right)_{2} \mathrm{Se}_{3}$, both values show a decrease in value to 0.03 $\mathrm{s}$, which can be attributed to much more surface dangling bonds, high crystal quality and preferential orientation. ${ }^{28,29}$ It is worth noting that these short response times are comparable to the very good results of some reported high performance photodetectors, ${ }^{30,31}$ further conforming the high potential application of $\mathrm{Sn}$-doped $\mathrm{Sb}_{2} \mathrm{Se}_{3}$ crystals as excellent photoelectric devices.

The optical absorption spectrum is one of the most effective tools for understanding and/or engineering the band structures of semiconductor materials. It is known that an appropriate band gap of the semiconductor plays a crucial role in fabricating high performance photoelectric devices. In this work, the optical absorption spectrum has been employed to characterize the as-synthesized $\left(\mathrm{Sn}_{x} \mathrm{Sb}_{1-x}\right)_{2} \mathrm{Se}_{3}$ crystals. And the absorption data were calculated from diffuse reflectance data using Kubelka-Munk equations: $F(R)=\alpha / \Lambda=(1-R)^{2} /(2 R)$, where $R$ is the reflectance, $\alpha$ and $\Lambda$ are the absorption and scattering coefficients, respectively. ${ }^{32}$ As shown in Fig. 7a, the onset of the absorption of $\mathrm{Sb}_{2} \mathrm{Se}_{3}$ crystals starts near $1200 \mathrm{~nm}$, and a shift toward a longer wavelength is observed after Sn doping. The higher absorption efficiency for the doped crystals in the NIR range is interesting for broad spectrum applications. Fig. 7b shows a plot of $[F(R) h \nu]^{2}$ versus energy $(h \nu)$; the direct band gap of $\mathrm{Sb}_{2} \mathrm{Se}_{3}$ is $1.17 \mathrm{eV}$, and then for the Sn-doped $\mathrm{Sb}_{2} \mathrm{Se}_{3}$ crystals, the band gap is similar and close to $1.10 \mathrm{eV}$. The dwindling band gaps of the doped $\left(\mathrm{Sn}_{x} \mathrm{Sb}_{1-x}\right)_{2} \mathrm{Se}_{3}$ crystals can be explained

\section{(a)}

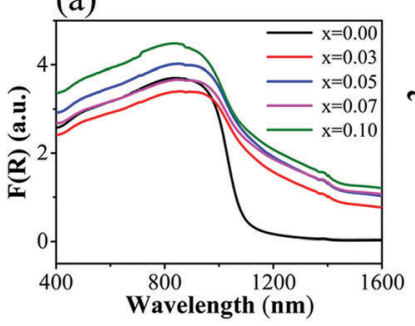

(b)

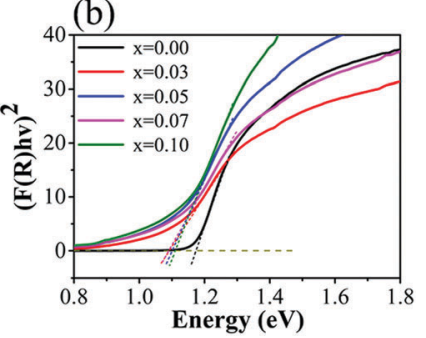

Fig. 7 (a) Optical absorption spectra (calculated from diffuse reflectance data) of the $\left(\mathrm{Sn}_{x} \mathrm{Sb}_{1-x}\right)_{2} \mathrm{Se}_{3}$ crystals $(x=0.00,0.03,0.05,0.07$ and 0.10$)(\mathrm{b}) \mathrm{a}$ plot of $[F(R) h \nu]^{2}$ vs. energy, from which direct band gap was obtained.

1 
1 by the cooperation of the Burstein-Moss shift and the renormalization effect. This mechanism exists in narrow band gap semiconductors with a specific doping level. ${ }^{33,34}$ Finally, it is worth noting that the band gap of the $\left(\mathrm{Sn}_{x} \mathrm{Sb}_{1-x}\right)_{2} \mathrm{Se}_{3}$ crystals is close to the best value for absorbing efficiently the solar spectrum, making them attractive candidates for photovoltaic application.

\section{Conclusions}

An effective melt-quenching method has been used to synthesize high-quality and variably doped $\left(\mathrm{Sn}_{x} \mathrm{Sb}_{1-x}\right)_{2} \mathrm{Se}_{3}$ crystals with the dopant $\mathrm{Sn}$ ratio $x$ ranging from 0.00 to 0.10 . The successful doping is verified by XRD, XPS and EDS results. With increasing $\mathrm{Sn}$ doping concentration, $\left(\mathrm{Sn}_{x} \mathrm{Sb}_{1-x}\right)_{2} \mathrm{Se}_{3}$ crystals exhibit an improvement of electrical conductivity with several orders of magnitude due to a much higher charge carrier concentration. Temperature dependent electrical conductivity displays a typical semiconductor characteristic and a decrease of activation energy is also confirmed with Sn incorporation. $\mathrm{Sn}$-Doped $\mathrm{Sb}_{2} \mathrm{Se}_{3}$ crystals are identified as p-type semiconductors according to the photoconductive characteristics. Notably, compared to pure $\mathrm{Sb}_{2} \mathrm{Se}_{3}$, the dark current density of a representative $\left(\mathrm{Sn}_{0.10} \mathrm{Sb}_{0.90}\right)_{2} \mathrm{Se}_{3}$ increased approximately 10 times and the photocurrent density increased approximately 14 times. In addition, it shows a short response/recovery time $(0.03 / 0.03 \mathrm{~s})$ and a reversible and stable photoresponse. Therefore, the $\mathrm{Sn}$-doped $\mathrm{Sb}_{2} \mathrm{Se}_{3}$ crystals have an optimal band gap, $(1.10 \mathrm{eV})$ which overlaps well with the solar spectrum, and excellent electrical conductivity and photoconductive properties, combined with their easy and relatively large-scale synthesis features. These properties confirm the high application potential of Sn-doped $\mathrm{Sb}_{2} \mathrm{Se}_{3}$ as highly efficient photoelectric devices.

\section{Conflicts of interest}

There are no conflicts to declare.

\section{Acknowledgements}

The authors gratefully acknowledge support for this research from the Program for Innovative Research Team in the University of Ministry of Education of China (IRT13R54), the Fundamental Research Funds for the Central Universities (2016FZA4007) and the Zhejiang Provincial Natural Science Foundation of China (LY16E020003). We also thank the Chinese Scholarship Council and the Eiffel Excellence Scholarship Programme for financial support.

\section{Notes and references}

1 L. Wang, D. B. Li, K. Li, C. Chen, H. X. Deng, L. Gao, Y. Zhao, F. Jiang, L. Li, F. Huang, Y. He, H. Song, G. Niu and J. Tang, Nat. Energy, 2017, 2, 17046.
2 G. X. Liang, X. H. Zhang, H. L. Ma, J. G. Hu, B. Fan, Z. K. Luo, Z. H. Zheng, J. T. Luo and P. Fan, Sol. Energy Mater. Sol. Cells, 2017, 160, 257-262.

3 T. Zhai, M. Ye, L. Li, X. Fang, M. Liao, Y. Li, Y. Koide, Y. Bando and D. Golberg, Adv. Mater., 2010, 22, 4530-4533.

4 Y. Q. Liu, M. Zhang, F. X. Wang and G. B. Pan, J. Mater. Chem. C, 2014, 2, 240-244.

5 T. Y. Ko, M. Shellaiah and K. W. Sun, Sci. Rep., 2016, 6, 35086 .

6 N. Hu, M. A. Cheney, Y. Hanifehpour, S. W. Joo and B.K. Min, J. Nanomater., 2017, 2017, 1-8.

7 Y. Zhou, L. Wang, S. Chen, S. Qin, X. Liu, J. Chen, D. J. Xue, M. Luo, Y. Cao, Y. Cheng, E. H. Sargent and J. Tang, Nat. Photonics, 2015, 9, 409-415.

8 Y. Zhou, M. Leng, Z. Xia, J. Zhong, H. Song, X. Liu, B. Yang, J. Zhang, J. Chen, K. Zhou, J. Han, Y. Cheng and J. Tang, Adv. Energy Mater., 2014, 4, 1301846.

9 R. Huang, J. Zhang, F. Wei, L. Shi, T. Kong and G. Cheng, Adv. Funct. Mater., 2014, 24, 3581-3586.

10 S. Chen, X. Qiao, F. Wang, Q. Luo, X. Zhang, X. Wan, Y. Xu and X. Fan, Nanoscale, 2016, 8, 2277-2283.

11 T. Zhai, L. Li, X. Wang, X. Fang, Y. Bando and D. Golberg, Adv. Funct. Mater., 2010, 20, 4233-4248.

12 D. Choi, Y. Jang, J. Lee, G. H. Jeong, D. Whang, S. W. Hwang, K. S. Cho and S. W. Kim, Sci. Rep., 2014, 4, 6714.

13 R. J. Mehta, C. Karthik, W. Jiang, B. Singh, Y. Shi, R. W. Siegel, T. Borca-Tasciuc and G. Ramanath, Nano Lett., 2010, 10, 4417-4422.

14 X. H. Zhang, Y. Xu, Q. H. Shen, B. Fan, X. S. Qiao, X. P. Fan, H. Yang, Q. Luo, L. Calvez, H. L. Ma, M. Cathelinaud and J. J. Simond, J. Mater. Chem. A, 2014, 2, 17099-17106.

15 D. Kong, K. J. Koski, J. J. Cha, S. S. Hong and Y. Cui, Nano Lett., 2013, 13, 632-636.

16 W. Tian, C. Zhi, T. Zhai, S. Chen, X. Wang, M. Liao, D. Golberg and Y. Bando, J. Mater. Chem., 2012, 22, 17984.

17 T. Zhai, Y. Ma, L. Li, X. Fang, M. Liao, Y. Koide, J. Yao, Y. Bando and D. Golberg, J. Mater. Chem., 2010, 20, 6630.

18 M. S. Iovu, S. D. Shutov, V. I. Arkhipov and G. J. Adriaenssens, J. Non-Cryst. Solids, 2002, 299, 1008-1012.

19 P. Barpanda, M. Ati, B. C. Melot, G. Rousse, J. N. Chotard, M. L. Doublet, M. T. Sougrati, S. A. Corr, J. C. Jumas and J. M. Tarascon, Nat. Mater., 2011, 10, 772-779.

20 C. H. Hu, M. H. Chiang, M. S. Hsieh, W. T. Lin, Y. S. Fu and T. F. Guo, CrystEngComm, 2014, 16, 1786-1792.

21 A. Y. Shenouda and H. K. Liu, J. Alloys Compd., 2009, 477, 498-503.

22 H. Song, T. Li, J. Zhang, Y. Zhou, J. Luo, C. Chen, B. Yang, C. Ge, Y. Wu and J. Tang, Adv. Mater., 2017, 29, 1700441.

23 X. Liu, D. Zhang, H. Li, L. Li and H. Yuan, Dalton Trans., 2017, 46, 2479-2484.

24 S. R. Culp, J. W. Simonson, S. J. Poon, V. Ponnambalam, J. Edwards and T. M. Tritt, Appl. Phys. Lett., 2008, 93, 022105.

25 S. Chen, X. Liu, X. Qiao, X. Wan, K. Shehzad, X. Zhang, Y. Xu and X. Fan, Small, 2017, 13, 1604033.

26 H. Zhang, X. Zhang, C. Liu, S. T. Lee and J. Jie, ACS Nano, 2016, 10, 5113-5122. 
127 R. L. Spray, K. J. McDonald and K. S. Choi, J. Phys. Chem. C, 2011, 115, 3497-3506.

28 K. Zhang, T. Luo, H. Chen, Z. Lou and G. Shen, J. Mater. Chem. C, 2017, 5, 3330-3335.

29 W. Zhou, Y. Peng, Y. Yin, Y. Zhou, Y. Zhang and D. Tang, AIP Adv., 2014, 4, 123005.

30 L. Zheng, K. Hu, F. Teng and X. Fang, Small, 2017, 34 Z. M. Gibbs, A. LaLonde and G. J. Snyder, New J. Phys., 2013, 13, 1602448.
31 L. Li, L. Gu, Z. Lou, Z. Fan and G. Shen, ACS Nano, 2017, 11, 4067-4076.

32 S. Wang and N. Ye, J. Am. Chem. Soc., 2011, 133, 11458-11461.

33 A. Walsh, J. L. F. Da Silva and S. H. Wei, Phys. Rev. B: Condens. Matter Mater. Phys., 2008, 78, 075211. 15, 075020 . 